\title{
BMJ
}

\section{Risk of death during and after opiate substitution treatment in primary care: prospective observational study in UK General Practice Research Database}

\author{
Rosie Cornish, statistician, ${ }^{1}$ John Macleod, professor in clinical epidemiology and primary care, ${ }^{1}$ John Strang, \\ professor in the psychiatry of the addictions, ${ }^{2}$ Peter Vickerman, senior lecturer in mathematical modelling, ${ }^{1,3}$ \\ Matt Hickman, professor in public health and epidemiology ${ }^{1}$
}

${ }^{1}$ School of Social and Community Medicine, University of Bristol Bristol BS8 2PS, UK

${ }^{2}$ National Addiction Centre, Institute of Psychiatry, King's

College London, London, UK

${ }^{3}$ London School of Hygiene and Tropical Medicine, London

Correspondence to: $\mathrm{M}$ Hickman matthew.hickman@bristol.ac.uk

Cite this as: $B M J$ 2010;341:c5475 doi:10.1136/bmj.c5475

\section{ABSTRACT}

Objective To investigate the effect of opiate substitution treatment at the beginning and end of treatment and according to duration of treatment.

Design Prospective cohort study.

Setting UK General Practice Research Database

Participants Primary care patients with a diagnosis of substance misuse prescribed methadone or buprenorphine during 1990-2005. 5577 patients with 267003 prescriptions for opiate substitution treatment followed-up (17 732 years) until one year after the expiry of their last prescription, the date of death before this time had elapsed, or the date of transfer away from the practice.

Mainoutcome measures Mortality rates and rate ratios comparing periods in and out of treatment adjusted for sex, age, calendar year, and comorbidity; standardised mortality ratios comparing opiate users' mortality with general population mortality rates.

Results Crude mortality rates were 0.7 per 100 person years on opiate substitution treatment and 1.3 per 100 person years off treatment; standardised mortality ratios were 5.3 (95\% confidence interval 4.0 to 6.8 ) on treatment and 10.9 (9.0 to 13.1) off treatment. Men using opiates had approximately twice the risk of death of women (morality rate ratio $2.0,1.4$ to 2.9 ). In the first two weeks of opiate substitution treatment the crude mortality rate was 1.7 per 100 person years: 3.1 (1.5 to 6.6) times higher (after adjustment for sex, age group, calendar period, and comorbidity) than the rate during the rest of time on treatment. The crude mortality rate was 4.8 per 100 person years in weeks 1-2 after treatment stopped, 4.3 in weeks 3-4, and 0.95 during the rest of time off treatment: 9 (5.4 to 14.9), 8 (4.7 to 13.7), and 1.9 (1.3 to 2.8 ) times higher than the baseline risk of mortality during treatment. Opiate substitution treatment has a greater than $85 \%$ chance of reducing overall mortality among opiate users if the average duration approaches or exceeds 12 months.

Conclusions Clinicians and patients should be aware of the increased mortality risk at the start of opiate substitution treatment and immediately after stopping treatment. Further research is needed to investigate the effect of average duration of opiate substitution treatment on drug related mortality.

\section{INTRODUCTION}

Opiate users have a high risk of death and contribute substantially to adult mortality. ${ }^{1}$ Systematic reviews estimate annual death rates of about $1 \%$, which is more than 10 times that of the general population and contributes more than $10 \%$ of adult mortality. ${ }^{2-4}$ In the United Kingdom and many other countries, most deaths among opiate users are due to overdose, and opiates are the most common cause of poisoning from controlled drugs in the population. ${ }^{5-8}$ Estimates of the prevalence of opiate use in the UK suggest 30fold increases between 1970 and $2000,{ }^{9}$ but more recent estimates are stable at around 250000 opiate users (approximately $0.8 \%$ of adults aged 15-64). ${ }^{1011}$

Good evidence shows that opiate substitution treatment, primarily with methadone and buprenorphine, is effective across a range of outcomes, including reducing all cause mortality, improving physical and mental health, and decreasing illicit drug use, criminal activity, and risk of HIV infection. ${ }^{12-19}$ In the UK, opiate substitution treatment is delivered mainly within primary care, often through shared care arrangements with specialist drug agencies..$^{2021}$ In the past decade, opiate prescription has more than doubled while the number of deaths involving methadone and police seizures of methadone have declined, coinciding in part with the introduction and roll-out of greater supervised consumption of opiate substitution treatment. ${ }^{722}$ However, the overall number of deaths from overdose of opiates has not decreased and targets to reduce deaths from overdose in England and Wales have not been met, despite an apparently stable population taking opiates and considerable investment in treatment. ${ }^{8}$

The risk of death during and after treatment seems to be non-uniform. Treatment guidelines and recent studies have suggested that the start of treatment (the first 28 days) may be associated with an increased risk of death. ${ }^{23-26}$ Furthermore, findings in two large cohorts 
in Italy and Australia have suggested that an excess risk of death exists in the period immediately after treatment ends. ${ }^{2427}$ We estimated the effect of opiate substitution treatment delivered in UK primary care on all cause mortality, investigated whether risk of death was elevated in the first few weeks of treatment and in the period immediately after treatment, and projected the likely impact of opiate substitution treatment on drug related mortality for different durations of treatment.

\section{METHODS}

The data for this study come from the General Practice Research Database (www.gprd.com), a large database of anonymised patients' records from more than 460 general practices in the UK. It collects data from 3.5 million patients, equivalent to around $5.5 \%$ of the UK's population. The study cohort consisted of patients who received at least one prescription for methadone or buprenorphine between 1 January 1990 and 31 December 2005 and who had a recorded diagnosis of substance misuse. We excluded patients if they were aged 60 or over when first prescribed buprenorphine or methadone, were prescribed injectable drugs, or were prescribed opiates specifically for pain. The prescription records contained details of each patient's unique database identification number, date of prescription, quantity prescribed, duration, and daily dose. We used each patient's identification number to link the prescription records to data on age and sex as well as to all other prescriptions dispensed to the patient during follow-up. If the quantity prescribed, duration, or daily dose was missing, we calculated it (where possible) from other prescription data. We defined patients as being "on treatment" until the expiry date of their last prescription unless they had a gap of less than 28 days between the expiry of one prescription and the issue date of the next. In such cases, we defined the patient as being "on treatment" during this gap. We chose this period because the prescription data within long term treatment episodes could be incomplete and we considered it unlikely that a patient would genuinely stop and restart treatment in general practice within a four week period. In addition, we examined the prescription data for evidence of other opiate substitution drugs and identified substantial numbers of patients with prescriptions for oral dihydrocodeine at levels prescribed for opiate substitution treatment, which has a history of use in general practice. ${ }^{28}$ Therefore, we included these dihydrocodeine prescriptions as opiate substitution treatment. We did not include patients prescribed only dihydrocodeine in the initial data extraction.

For each treatment episode, we determined whether the patient received at least one daily dose of methadone, buprenorphine, or dihydrocodeine on or above the recommended maintenance thresholds of $60 \mathrm{mg}$, $12 \mathrm{mg}$, and $600 \mathrm{mg} .{ }^{2628}$ For patients on methadone or buprenorphine, we defined "planned discharge" on the basis of a tapering dose towards the end of the prescribing period with last prescription being for methadone $10 \mathrm{mg}$ or less or buprenorphine $4 \mathrm{mg}$ or less. We calculated a comorbidity score as the total number of prescriptions for drugs other than opiate substitution treatment during each year of follow-up; this method is similar in performance to more complex comorbidity measures that until very recently were unavailable for the General Practice Research Database. ${ }^{2930}$

\section{Statistical analysis}

The outcome measure was all cause mortality. A patient's follow-up ceased either one year after the expiry of their last prescription for opiate substitution treatment, on the date of death before this time had elapsed, or on the date of transfer away from the practice (while on opiate substitution treatment or in the 12 month period after treatment). Patients transferred away or lost to follow-up contributed their exposure on or off treatment until the date of transfer. Several of the covariates varied with time. Time on or off treatment, including whether this was in the first two or four weeks, was the key exposure; we also divided each patient's follow-up into five year age groups and according to calendar year, treatment episode, and comorbidity score. We calculated crude mortality rates by dividing the total number of deaths in a particular exposure subgroup by the total amount of follow-up in this group. We used Poisson regression to estimate mortality rate ratios comparing periods in and out of treatment, adjusted for potential confounders (sex, age group, calendar period, comorbidity score, treatment episode, and episodes with one opiate substitution treatment at or above recommended levels for maintenance). We calculated indirect standardised mortality ratios on the basis of 1998 sex specific death rates in England and Wales for five year age groups. We used Stata version 10.0 for analyses.

\section{Sensitivity and secondary analyses}

We fitted an interaction to test for differences in the risk of death at different treatment periods between methadone and buprenorphine. In addition, we fitted an interaction to test whether a difference existed in the risk of death in the first 28 days "off treatment" for patients with evidence of tapered prescribing before treatment ceased. We may have misclassified time on and off treatment (in our attempt to allow for missing information on prescriptions) and introduced biasfor example, if occasions existed when patients were genuinely off treatment during a gap of less than 28 days between prescriptions. Therefore, we did a sensitivity analysis by restricting the analysis to each patient's final treatment episode. We also restricted the analysis to patients with no prescriptions for oral dihydrocodeine to test whether the findings were the same for patients receiving only methadone or buprenorphine. Finally, we estimated the probability that opiate substitution treatment reduces average mortality for patients exposed to different durations of treatment compared with if they had been unexposed (see web appendix for methods). 


\section{RESULTS}

Sample

The original study cohort included 6252 patients aged 16 to 59 years receiving opiate substitution treatment with either methadone or buprenorphine (web appendix fig A). We excluded the following patients: 74 prescribed buprenorphine patches; 19 whose prescriptions specified "for pain;" 152 who received injectable methadone or buprenorphine; 430 patients (5983 prescriptions) with insufficient data on duration, quantity, and daily dose to calculate periods on and off treatment. This left 5577 (89\%) patients with 231272 prescriptions for methadone or buprenorphine. We added 35731 prescriptions for oral dihydrocodeine prescriptions, making a final total of 267003 prescriptions.

Table 1 shows the characteristics of the sample. More than half of the patients were prescribed only methadone, $8 \%$ were prescribed only buprenorphine, and $35 \%$ received a combination of different opiate substitution treatments (including dihydrocodeine). The median length of follow-up was 2.0 years, and patients contributed a total of 17732 person years of follow-up: 8940 person years on treatment and 8792 off treatment. Between 1990 and 2005, 1647 (30\%) patients transferred out of their practices: 743 during opiate substitution treatment and 904 in the 12 months after treatment ( $8 \%$ and $10 \%$ of the person years of follow-up). Patients who transferred out during the study were more likely to be women (34\% v 29\%), to be younger (65\% v 55\% aged under 30$)$, and to have less comorbidity (58\% v $63 \%$ with lowest comorbid score).

More than half of the patients were aged under 30 at the start of treatment, and $3867(69 \%)$ were men. Altogether, 13593 treatment episodes occurred (a median of two per patient), with a median duration of 70 days ( 2.3 months) and a mean duration of 240 days (7.9 months); 36\% (2033) of patients had at least one treatment episode lasting 12 months or more; and the mean length of treatment episode was longer for methadone than for buprenorphine (245 v 181 days, $\mathrm{P}<0.001$, unpaired $t$ test). Daily dose was available for $13118(97 \%)$ of the 13593 treatment episodes; of these, $3261(24 \%)$ episodes included at least one daily dose above the recommended maintenance threshold. A tapered dose at the end of the prescribed period was recorded for $10 \%$ of methadone episodes and $46 \%$ of buprenorphine episodes.

Effect of opiate substitution treatment on mortality A total of $178(3 \%)$ patients died either on treatment or within a year of their last prescription; of these, 62 $(35 \%)$ died while on treatment. The crude mortality rate off treatment was almost double that on treatment (1.3 $v 0.7$ per 100 person years), and after adjustment (for age, sex, calendar period, and comorbidity) the mortality rate ratio was more than twice as high (2.3, $95 \%$ confidence interval 1.7 to 3.1 ). The standardised mortality ratio comparing death rates among study patients with the population of England and Wales was 5.3 (4.0 to 6.8 ) while on treatment and 10.9 (9.0 to 13.1) off treatment. As mortality in young adults is generally almost twice as high among men as in women (mortality rate ratio 2.0, 1.4 to 2.9 ) (table 2), the standardised mortality ratio was similar for men and women (5.0 and 6.4 on treatment and 11.0 and 10.4 off treatment).

Table 2 shows the main findings. Mortality increased with age and was positively associated with comorbidity score. Mortality was lower in 2005-6 than in 1990-4, although after adjustment insufficient evidence existed to suggest that mortality had declined over time. Mortality was unrelated to treatment

Table 1|Characteristics of patients and treatment episodes

\begin{tabular}{lc} 
Characteristics & No (\%)* \\
Patients & $(\mathrm{n}=5577)$ \\
\hline Male sex & $3867(69)$ \\
\hline Age (years) at start of treatment: & $260(5)$ \\
\hline$\langle 20$ & $2950(53)$ \\
\hline $20-29$ & $1784(32)$ \\
\hline $30-39$ & $471(8)$ \\
\hline $40-49$ & $112(2)$ \\
\hline$\geq 50$ & 281 days \\
\hline Total length of time on treatment: & 1 day to 15.9 years \\
\hline Median & $585(787)$ days \\
\hline Range & $3159(57)$ \\
\hline Mean (SD) & $456(8)$ \\
\hline Treatment received: & $482(9)$ \\
\hline Methadone only & $1045(19)$ \\
\hline Buprenorphine only & $227(4)$ \\
\hline Methadone and buprenorphine & $208(4)$ \\
\hline Methadone and dihydrocodeine & \\
\hline Buprenorphine and dihydrocodeine & \\
\hline $\begin{array}{l}\text { Methadone, buprenorphine, and } \\
\text { dihydrocodeine }\end{array}$ & \\
\hline
\end{tabular}

No of treatment episodes:

\begin{tabular}{lc}
\hline 1 & $2509(45)$ \\
\hline 2 & $1261(23)$ \\
\hline 3 & $693(12)$ \\
\hline $5-9$ & $457(8)$ \\
\hline$\geq 10$ & $565(10)$ \\
\hline Episodes & $92(2)$ \\
\hline$\leq 1 /$ month & $7781(57)$ \\
\hline$>1 /$ month to $\leq 1 /$ week & $3531(26)$ \\
\hline >1/week & $2281(17)$ \\
\hline At least one dose on/above recommended & $3261 / 13118(25)$ \\
\hline threshold $\neq$ & \\
\hline Last dose $\leq 10$ mg methadone & $709 / 7064(10)$ \\
\hline Last dose $\leq 4$ mg buprenorphine & $607 / 1332(46)$ \\
\hline Length of treatment episodes: & 70 days \\
\hline Median & $240(440)$ days \\
\hline Range & \\
\hline Mean (SD) & \\
\hline
\end{tabular}

*Unless otherwise specified.

†As measured by number of non-opiate substitution prescriptions received by patient in calendar year.

$\ddagger 60 \mathrm{mg}$ for methadone, $12 \mathrm{mg}$ for buprenorphine, and $600 \mathrm{mg}$ for dihydrocodeine. 
Table 2 |Crude mortalities and mortality rate ratios for all cause mortality

\begin{tabular}{|c|c|c|c|c|c|c|c|}
\hline \multirow[b]{2}{*}{ Variable } & \multirow[b]{2}{*}{ Deaths } & \multirow[b]{2}{*}{ Person years } & \multirow[b]{2}{*}{$\begin{array}{l}\text { Mortality/100 } \\
\text { person years }\end{array}$} & \multicolumn{2}{|c|}{ Crude analysis } & \multicolumn{2}{|c|}{ Adjusted analysis* } \\
\hline & & & & $\begin{array}{l}\text { Mortality rate ratio } \\
\qquad(95 \% \mathrm{Cl})\end{array}$ & $P$ value & $\begin{array}{l}\text { Mortality rate ratio } \\
\qquad(95 \% \mathrm{Cl})\end{array}$ & $P$ value \\
\hline Overall on treatment & 62 & 8939.7 & 0.69 & 1.00 & \multirow{2}{*}{$<0.001$} & 1.00 & \multirow{2}{*}{$<0.001$} \\
\hline Overall off treatment & 116 & 8791.8 & 1.32 & 1.90 (1.40 to 2.59$)$ & & 2.29 (1.67 to 3.14$)$ & \\
\hline \multicolumn{8}{|l|}{ Period: } \\
\hline Weeks $1-2$ of treatment & 8 & 471.5 & 1.70 & $2.80(1.33$ to 5.91$)$ & \multirow{6}{*}{$<0.001$} & 3.11 (1.47 to 6.59$)$ & \multirow{6}{*}{$<0.001$} \\
\hline Weeks 3-4 of treatment & 5 & 378.7 & 1.32 & $2.18(0.87$ to 5.47$)$ & & $2.38(0.95$ to 5.99$)$ & \\
\hline Remainder of time on treatment & 49 & 8089.4 & 0.61 & 1.00 & & 1.00 & \\
\hline Weeks $1-2$ off treatment & 22 & 458.0 & 4.80 & $7.93(4.80$ to 13.12$)$ & & 9.01 (5.43 to 14.90$)$ & \\
\hline Weeks 3-4 off treatment & 19 & 446.6 & 4.25 & $7.02(4.11$ to 11.93$)$ & & $8.01(4.70$ to 13.66$)$ & \\
\hline Remainder of time off treatment & 75 & 7887.2 & 0.95 & $1.57(1.10$ to 2.25$)$ & & $1.91(1.32$ to 2.76$)$ & \\
\hline \multicolumn{8}{|l|}{ Sex: } \\
\hline Female & 36 & 5270.2 & 0.68 & 1.00 & \multirow{2}{*}{0.004} & 1.00 & \multirow{2}{*}{$<0.001$} \\
\hline Male & 142 & 12461.2 & 1.14 & 1.67 (1.16 to 2.40$)$ & & $1.97(1.36$ to 2.85$)$ & \\
\hline \multicolumn{8}{|l|}{ Age (years): } \\
\hline$\ll 30$ & 53 & 7158.0 & 0.74 & 1.00 & \multirow{4}{*}{$<0.001$} & 1.00 & \multirow{4}{*}{$<0.001$} \\
\hline 30-39 & 64 & 7187.5 & 0.89 & $1.20(0.84$ to 1.73$)$ & & 1.24 (0.86 to 1.79$)$ & \\
\hline $40-49$ & 37 & 2650.3 & 1.40 & 1.89 (1.24 to 2.87$)$ & & 1.85 (1.20 to 2.85$)$ & \\
\hline$\geq 50$ & 24 & 735.6 & 3.26 & $4.41(2.72$ to 7.14$)$ & & $4.12(2.47$ to 6.86$)$ & \\
\hline \multicolumn{8}{|l|}{ Calendar year: } \\
\hline $1990-4$ & 23 & 1326.4 & 1.73 & 1.00 & \multirow{4}{*}{0.05} & 1.00 & \multirow{4}{*}{0.1} \\
\hline $1995-9$ & 52 & 4806.5 & 1.08 & 0.70 (0.38 to 1.02$)$ & & 0.65 (0.40 to 1.06$)$ & \\
\hline $2000-4$ & 75 & 8405.7 & 0.89 & $0.56(0.32$ to 0.82$)$ & & $0.54(0.34$ to 0.87$)$ & \\
\hline $2005-6$ & 28 & 3192.9 & 0.88 & $0.56(0.29$ to 0.88$)$ & & $0.54(0.31$ to 0.95$)$ & \\
\hline \multicolumn{8}{|l|}{ Comorbidity score†: } \\
\hline$\leq 1 /$ month & 70 & 10003.8 & 0.70 & 1.00 & \multirow{3}{*}{$<0.001$} & 1.00 & \multirow{3}{*}{$<0.001$} \\
\hline$>1 /$ month to $\leq 1 /$ week & 56 & 4630.9 & 1.21 & $1.73(1.22$ to 2.46$)$ & & 1.78 (1.24 to 2.55$)$ & \\
\hline s1/week & 52 & 3096.7 & 1.68 & 2.40 (1.68 to 3.44$)$ & & 2.37 (1.61 to 3.48$)$ & \\
\hline \multicolumn{8}{|l|}{ Treatment episode: } \\
\hline First & 80 & 7925.9 & 1.01 & 1.00 & \multirow{4}{*}{0.7} & \multirow{4}{*}{\multicolumn{2}{|c|}{ Not included }} \\
\hline Second & 35 & 4086.6 & 0.86 & $0.85(0.57$ to 1.26$)$ & & & \\
\hline Third or fourth & 39 & 3577.8 & 1.09 & 1.08 (0.74 to 1.58$)$ & & & \\
\hline Fifth or above & 24 & 2141.2 & 1.12 & $1.11(0.70$ to 1.75$)$ & & & \\
\hline \multicolumn{8}{|l|}{ At least one dose on/above thresholdf: } \\
\hline No & 115 & 11546.9 & 1.00 & 1.00 & & Noti & \\
\hline Yes & 53 & 5646.7 & 0.94 & 0.94 (0.68 to 1.30$)$ & 0.7 & Not Ine & \\
\hline
\end{tabular}

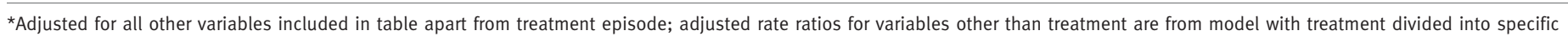
periods.

†As measured by number of non-opiate substitution prescriptions received by patient in calendar year.

‡Daily dose missing for 475 treatment episodes.

episode and whether the treatment episode included at least one dose on or above the recommended maintenance thresholds.

After the first four weeks of treatment, the mortality rate for the rest of time on treatment was 0.6 per 100 person years (table 2). The mortality rate was raised in the first four weeks: 1.7 per 100 person years in the first two weeks and 1.3 per 100 person years in weeks three to four, which after adjustment was 3.1 (1.5 to 6.6) and $2.4(0.95$ to 6.0$)$ times higher than the mortality in the rest of time on treatment. Mortality was also raised substantially in the period immediately after treatment, at 4.8 per 100 person years in the first two weeks and 4.3 per 100 person years in weeks three to four, falling thereafter to just under 1 per 100 person years. After adjustment, mortality was more than eight times higher in the first month after treatment stopped and almost twice as high in the remainder of time off treatment compared with the rate during the stable period (from one month onwards) on treatment. The adjusted death rate in the first month after treatment stopped was more than four times higher (mortality rate ratio $4.20,2.53$ to 6.96 ) than in the subsequent period off treatment.

\section{Sensitivity and secondary analyses}

We found no difference in the risk of death immediately after treatment between treatment episodes with and without evidence of tapered prescribing (that is, planned discharge). The mortality rate ratios in first 28 days off treatment were 8.75 (2.34 to 32.75$)$ with evidence of tapered prescribing and 8.27 (4.78 to 14.30) without such evidence (interaction test: $\left.\chi^{2}=0.32, \mathrm{df}=2, \mathrm{P}=0.9\right)$. 
Table $3 \mid$ Mortalities and rate ratios in different periods on/off treatment for buprenorphine and methadone separately (excluding periods when patient switched during first 28 days of treatment)

\begin{tabular}{|c|c|c|c|c|c|}
\hline Drug & Period & Deaths & Person years & $\begin{array}{l}\text { Mortality } / 100 \\
\text { person years }\end{array}$ & $\begin{array}{l}\text { Adjusted mortality rate ratio } \\
\qquad(95 \% \mathrm{Cl})\end{array}$ \\
\hline \multirow{4}{*}{ Methadone } & Days $1-28$ on treatment & 7 & 464.5 & 1.51 & 2.84 (1.14 to 7.04$)$ \\
\hline & Remainder on treatment & 23 & 4664.0 & 0.49 & 1.00 \\
\hline & Days $1-28$ off treatment & 21 & 479.0 & 4.38 & 9.47 (5.13 to 17.47$)$ \\
\hline & Remainder off treatment & 50 & 3809.2 & 1.31 & 2.99 (1.78 to 5.04$)$ \\
\hline \multirow{4}{*}{ Buprenorphine } & Days $1-28$ on treatment & 1 & 80.5 & 1.24 & $2.49(0.33$ to 18.51$)$ \\
\hline & Remainder on treatment & 6 & 659.2 & 0.91 & 1.64 (0.65 to 4.09$)$ \\
\hline & Days $1-28$ off treatment & 7 & 87.5 & 8.00 & 15.68 (6.66 to 36.92$)$ \\
\hline & Remainder off treatment & 3 & 663.1 & 0.45 & 0.96 (0.29 to 3.23$)$ \\
\hline \multirow{2}{*}{ Methadone } & Overall on treatment & 30 & 5128.5 & 058 & 1.00 \\
\hline & Overall off treatment & 71 & 4288.2 & 1.66 & 3.27 (2.08 to 5.14$)$ \\
\hline \multirow{2}{*}{ Buprenorphine } & Overall on treatment & 7 & 739.7 & 0.95 & 1.49 (0.64 to 3.45$)$ \\
\hline & Overall off treatment & 10 & 750.6 & 1.33 & 2.45 (1.18 to 5.07$)$ \\
\hline
\end{tabular}

We found no evidence of any difference in the risk of death between buprenorphine and methadone when we compared the whole period on and off treatment (table 3). However, we found a few differences in the crude mortality rates at different periods on and off treatment between methadone and buprenorphine but no or weak evidence of an interaction $\left(\chi^{2}=7.2\right.$, $\mathrm{df}=3, \mathrm{P}=0.06$ ) (table 3 ). Specifically, we found no evidence that the risk of death in the first 28 days on or first 28 days off treatment differed between methadone and buprenorphine. For example, the adjusted mortality rate ratio was 2.8 (1.1 to 7.0$)$ and 2.5 (0.3 to 18.5$)$ for the first 28 days on methadone or buprenorphine and 9.5 (5.1 to 17.5$)$ and 15.7 (6.7 to 36.9$)$ for the first 28 days off methadone or buprenorphine, compared with the risk of death on methadone after the first 28 days of treatment.

Restricting the analysis to the last treatment episode for each patient (where the date of last treatment was clear) gave similar results to those in table 2 (see web appendix table A). Excluding patients who had ever had a prescription for dihydrocodeine $(n=1480$ patients) did not alter the overall findings (web appendix table B).

Figure 1 shows the adjusted mortality rate ratios at different critical periods during and immediately after treatment compared with the general mortality when out of treatment. Figure 2 compares the average risk of death while on opiate substitution treatment with that while not on opiate substitution treatment by duration of treatment. Figure 3 estimates the probability that opiate substitution treatment may reduce overall mortality among the opiate dependent population for different average durations of treatment. Given the differential mortality rates shown in fig 1 , treatment of comparatively short duration (20 or 30 weeks) seems not to reduce the overall risk of death (less than 25\% chance; see fig 2 and fig 3). At an average of 40 weeks' duration of treatment, the chance that treatment reduces overall mortality was $65 \%$; at longer treatment durations approaching or exceeding a year, opiate substitution treatment is highly likely (more than $85 \%$ chance) to reduce the overall mortality in opiate users and lead to a decline in the number of drug related deaths.

\section{DISCUSSION}

Compared with the general population, opiate users in this study had a substantially higher risk of death. The overall risk of death during opiate substitution treatment was lower than the risk of death out of treatment. Patients started on opiate substitution treatment had a twofold to threefold higher risk of death in the first 14 and 28 days of treatment compared with the risk during the rest of their time on treatment. The risk of death increased eightfold to ninefold in the month immediately after the end of opiate substitution treatment. We found no strong evidence that these effects varied according to the type of treatment (methadone or buprenorphine), whether the dose was within recommended treatment thresholds, or whether cessation of treatment seemed to have been planned (evidence of a reduced dose before cessation) or unplanned. We hypothesise that the net benefit of opiate substitution treatment on overall mortality may relate to the average duration of treatment.

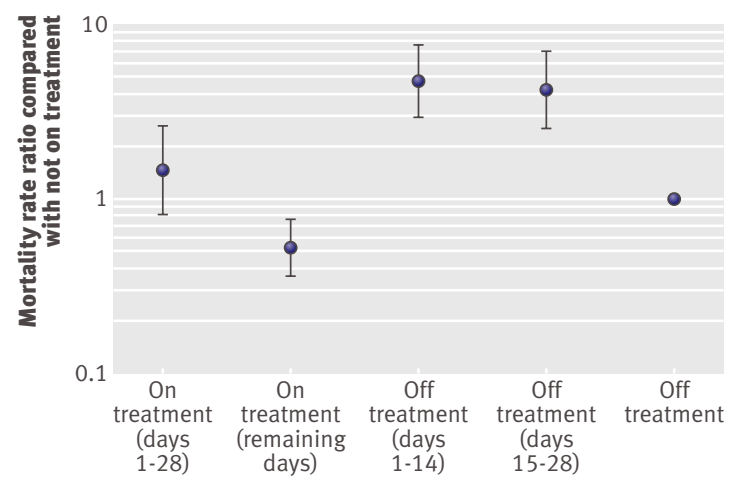

Fig 1| Adjusted risk of death, compared with not being on treatment, during and after opiate substitution treatment 


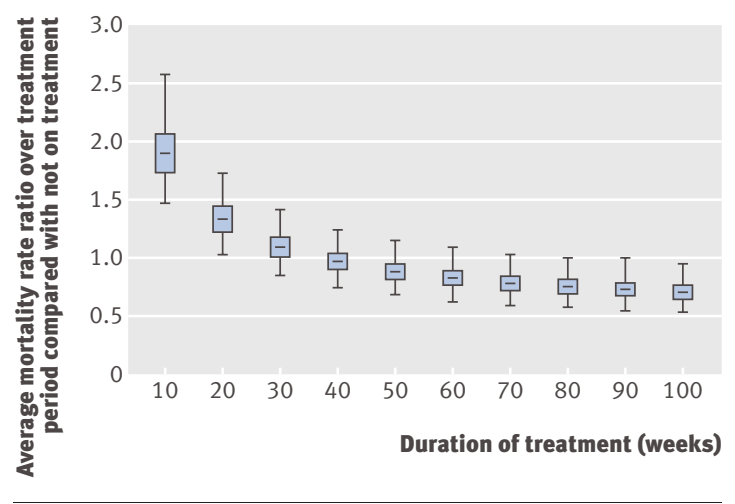

Fig 2 | Comparison of risk of death in patients exposed or not exposed to opiate substitution treatment (OST) by duration of treatment. Boxes are interquartile ranges (with median); lines are $95 \%$ confidence intervals

\section{Strengths and weaknesses}

Previous large scale studies of drug related mortality in the UK have not investigated the effect of opiate substitution treatment. ${ }^{31} 32$ The General Practice Research Database covers approximately $5 \%$ of the population in general practice and provides a large, reasonably representative sample of patients taking opiate substitution treatment. Large scale observational studies are needed because although the risk of death in the first four weeks of starting or stopping treatment is comparatively high, it is transient and unlikely to be detected in any general practice or even specialist drug clinic or by any randomised controlled trial. For instance, the mortality in the first four weeks of opiate substitution treatment was equivalent to 1 per 1000 treatments, which no general practice and very few specialist drug clinics will achieve in one year (and no trials in this area are likely to have sufficient power to detect differences in such an outcome).

Our study has several weaknesses and potential biases. Firstly, classification of time on and off treatment was complicated by occasional gaps in the information on doses, duration, or quantity prescribed, which may have led to an underestimate or overestimate of the mortality on or off treatment and therefore have diluted or exaggerated the difference between them. However, the findings were similar in a partial sensitivity test in which we restricted the analysis to the last treatment episode, and the data are consistent with other studies. ${ }^{192427}$

Secondly, patients who transferred out of the database (which in our study was about $10 \%$ per year) had no active follow-up, and patients' opiate use after leaving opiate substitution treatment was not followed up. Differences existed in the characteristics of patients who transferred and those who completed follow-up. We assumed, therefore, that the mortality of patients who transferred was the same as for those who remained in their practice, given adjustment for the characteristics that were recorded. However, other factors or unrecorded confounders may be associated with both mortality and transferring from the study practice (loss to follow-up) that could be a source of bias. In addition, some patients who left treatment will have stopped their opiate use (and therefore have been at substantially less risk of death than were patients who relapsed). For this reason, we restricted follow-up to 12 months after treatment, as any misclassification of patients (and person years) at risk is likely to be small because the rate of cessation/long term abstinence is low ${ }^{3334}$; however, this bias may contribute to a dilution in the risk ratio of mortality for opiate users in and out of treatment.

Thirdly, we had limited covariates for which we could adjust the analyses. For example, we adjusted for an index of comorbidity, but it was not an ideal measure and it was not specific to opiate users. ${ }^{29}$ More importantly, we had no factors that could measure the quality or intensity of the intervention, which may have a bearing on both the risk of death at starting opiate substitution treatment and the likelihood of relapse and death in the period immediately after treatment. ${ }^{2635}$

Fourthly, the outcome of our study was all cause mortality, as we had no information on specific causes of death. Although previous studies in the UK have shown that most deaths among active opiate users will be due to overdose, some studies have shown a greater difference in the risk of overdose during and after treatment than for all cause mortality, which we would not have detected. ${ }^{27}$

Finally, our comparisons of the risk of death in exposed and unexposed populations by duration of opiate substitution treatment are calculated effects and not directly observed effects. The model estimates should be taken as evidence to inform (rather than test) our hypothesis, especially given the limited number of confounders and that these were secondary analyses. Nevertheless, no trial is likely to have sufficient power to measure the effect of duration of opiate substitution treatment on overall risk of death, so future analyses must inevitably be derived from observational cohorts.

\section{Findings in relation to other studies}

The overall risk of death, standardised mortality ratios, and overall difference in mortality between time on and off treatment for opiate users in UK primary care in this study are consistent with international literature. ${ }^{46}$ As previously found in the UK, the risk

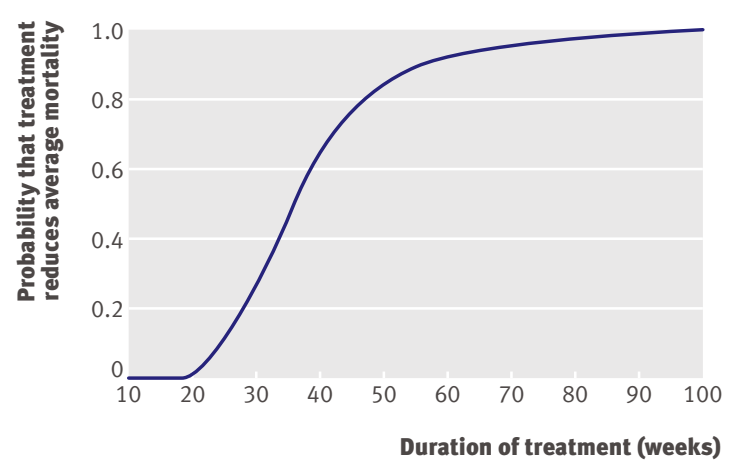

Fig 3 Probability that opiate substitution treatment (OST) reduces overall mortality for different durations of treatment 


\section{WHAT IS ALREADY KNOWN ON THIS TOPIC}

Risk of death is reduced during opiate substitution treatment

The risk of death may be raised at the start of opiate substitution treatment, and two cohorts report considerable excess mortality in the first few weeks after stopping treatment

\section{WHAT THIS STUDY ADDS}

Patients started on opiate substitution treatment had a twofold to threefold higher risk of death in the first 14 and 28 days of treatment compared with the rest of their time on treatment

The risk of death was eightfold to ninefold higher in the month immediately after treatment stopped

The effect of opiate substitution treatment on overall drug related mortality may relate to the average duration of treatment in the population

of death was higher among men using opiates than in women, which is primarily because men have a higher risk of overdose than do women; it also means that, as mortality among young adult men in the general population is higher than in women, the standardised mortality ratios for men and women using opiates were similar. ${ }^{37}$ Some other countries with higher rates of infection related deaths have a similar crude mortality rate in men and women using opiates and therefore a higher standardised mortality ratio in women. ${ }^{24}$

The difference in mortality between opiate users in and out of treatment is stark and well known, but fewer studies have investigated the risk of death at the start of or immediately after treatment. ${ }^{13192535}$ Studies in Australia and Holland also report a twofold risk in the first month of treatment ${ }^{2324}$; the study in Australia showed that these deaths were primarily due to overdose and other injury. ${ }^{24}$ In contrast, we found a substantially larger difference in mortality in the first month after treatment stopped than did either the study in Australia (mortality rate ratio approximately 3 ) or the one in Holland (mortality rate ratio approximately 1 ). Our findings were similar to those of a study in Italy, which reported a 10-fold higher risk of overdose in the first month after treatment stopped compared with the risk during treatment. ${ }^{27}$ Furthermore, the fourfold difference in mortality between the first month after the end of treatment and the rest of the time off treatment is similar to the excess risk of death in the first four weeks after release from prison in the UK compared with the risk of death during the rest of the year after prison. ${ }^{38}$ Long term follow-up of injecting drug users from a single primary care centre suggests that survival is substantially improved with each year of exposure to opiate substitution treatment ${ }^{34}$ as we estimate in figure 2. In addition, McCowan and colleagues showed that overall survival was unrelated to recommended levels of methadone prescription. ${ }^{39}$

\section{Implications for policy and practice}

Our study could not test mechanisms, so any inference in this regard must be cautious. However, we know that death from overdose of opiates is caused by respiratory depression, that the risk of overdose is increased at times when tolerance of opiate has temporarily fallen after voluntary or involuntary reductions in use,$^{40}$ and that relapse rates after treatment or abstinence from opiate use are high. ${ }^{1541}$ Therefore, the first few occasions of opiate use pose the greatest risk of precipitating fatal overdose before tolerance has been re-established. Induction on to opiate substitution treatment, especially with methadone, also poses risks in terms of overdose if the initial dose is too high or if patients continue to use non-prescribed opiates, and the change may be a cause of mental distress. ${ }^{2426}$ Closer supervision of induction of treatment alongside the development of more effective methods of preventing relapse may mitigate these effects, and further research should consider these questions. The identification and evaluation of better management and practice of opiate substitution treatment will be complex and require large scale studies. Ongoing monitoring of the impact of opiate substitution treatment on mortality in the UK and other countries is warranted, particularly investigation of specific causes of death. As most trials are severely underpowered to investigate risk of death, establishing the extent to which time to "relapse" or frequency of use can be a surrogate outcome or biomarker of mortality will be very important.

Finally, provision of opiate substitution treatment has substantially increased in the UK, ${ }^{7}$ but the total number of deaths due to overdose of opiates has remained stable and national targets to reduce their number have not been met. ${ }^{6}$ We hypothesise that the raised risk of death in the first month of treatment and especially in the month after the end of treatment may negate any protective effect of opiate substitution treatment, unless treatment is prolonged. In our study, the mean duration of treatment was 34 weeks which, as figure 3 suggests, may not necessarily lead to a decline in the overall number of deaths. Further research is needed to test this hypothesis and investigate the effect of average duration of opiate substitution treatment on drug related mortality.

This study is based in part on data from the Full Feature General Practice Research Database obtained under licence from the UK Medicines and Healthcare Products Regulatory Agency (MHRA). We acknowledge the help and support of MHRA staff in taking forward our analyses. However, the interpretation and conclusions contained in this study are those of the authors alone.

Contributors: $\mathrm{MH}$ conceived the study and obtained funding. RC analysed GPRD data and did the main analyses; PV did additional calculations. All authors contributed to interpretation of the findings and writing of the manuscript. $\mathrm{MH}$ is the guarantor.

Funding: Access to and permission to use the GPRD (protocol 06_058) was funded through the Medical Research Council's licence agreement with the MHRA. RC was part funded by a grant from the National Institute of Health Research (NIHR) for the Centre for Research on Drugs and Health Behaviour. At the time they did this work, JM and MH were supported by career scientist fellowship awards from the NIHR. PV is supported by an MRC new investigator award.

Competing interests: None declared.

Data sharing: No additional data available (permission for use of the original data can be sought from the MHRA).

1 Advisory Council on the Misuse of Drugs. Reducing drug-related deaths: a report by the Advisory Council on the Misuse of Drugs. ACMD, Home Office, 2000. 
2 Bargagli AM, Hickman M, Davoli M, Perucci CA, Schifano P, Buster M, et al. Drug-related mortality and its impact on adult mortality in eight European countries. Eur J Public Health 2006;16:198-202.

3 Gossop M, Stewart D, Treacy S, Marsden J. A prospective study of mortality among drug misusers during a 4-year period after seeking treatment. Addiction 2002;97:39-47.

4 Degenhardt L, Bucello C, Mathers B, Briegleb C, Ali H, Hickman M, et al. Mortality among regular or dependent users of heroin and othe opioids: a systematic review and meta-analysis of cohort studies. Addiction [forthcoming].

5 Gossop M, Browne N, Stewart D, Marsden J. Alcohol use outcomes and heavy drinking at 4-5 years among a treatment sample of drug misusers. J Subst Abuse Treat 2003;25:135-43.

6 Morgan O, Vicente J, Griffiths P, Hickman M. Trends in overdose deaths from drug misuse in Europe: what do the data tell us? Addiction 2008;103:699-700.

7 Morgan O, Griffiths C, Hickman M. Association between availability of heroin and methadone and fatal poisoning in England and Wales 1993-2004. Int J Epidemiol 2006;35:1579-85.

8 Morgan O, Griffiths C, Toson B, Rooney C, Majeed A, Hickman M. Trends in deaths related to drug misuse in England and Wales, 1993 2004. Health Stat Q 2006;31:23-7.

9 De Angelis D, Hickman M, Yang SY. Estimating long-term trends in the incidence and prevalence of opiate use/injecting drug use and the number of former users: back-calculation methods and opiate overdose deaths. Am J Epidemiol 2004;160:994-1004.

10 Hay G, Gannon M, Macdougall J, Eastwood C, Williams K, Millar T. Capture-recapture and anchored prevalence estimation of injecting drug users in England: national and regional estimates. Stat Methods Med Res 2009;18:323-39.

11 De Angelis D, Sweeting M, Ades AE, Hickman M, Hope V, Ramsay M. An evidence synthesis approach to estimating hepatitis $C$ prevalence in England and Wales. Stat Methods Med Res 2009;18:361-79.

12 Ward J, Hall W, Mattick RP. Role of maintenance treatment in opioid dependence. Lancet 1999;353:221-6.

13 Mattick RP, Breen C, Kimber J, Davoli M. Methadone maintenance therapy versus no opioid replacement therapy for opioid dependence. Cochrane Database Syst Rev 2009;3:CD002209.

14 Sporer KA. Strategies for preventing heroin overdose. BMJ 2003;326:442-4

15 Amato L, Davoli M, Perucci CA, Ferri M, Faggiano F, Mattick RP. An overview of systematic reviews of the effectiveness of opiate maintenance therapies: available evidence to inform clinical practice and research. J Subst Abuse Treat 2005;28:321-9.

16 Gowing L, Farrell M, Sullivan LE, Ali R. Substitution treatment of injecting opioid users for prevention of HIV infection. Cochrane Database Syst Rev 2007;4:CD004145.

17 Mattick RP, Kimber J, Breen C, Davoli M. Buprenorphine maintenance versus placebo or methadone maintenance for opioid dependence. Cochrane Database Syst Rev 2008;2:CD002207.

18 Bell J, Trinh L, Butler B, Randall D, Rubin G. Comparing retention in treatment and mortality in people after initial entry to methadone and buprenorphine treatment. Addiction 2009;104:1193-200.

19 Fugelstad A, Stenbacka M, Leifman A, Nylander M, Thiblin I. Methadone maintenance treatment: the balance between life-saving treatment and fatal poisonings. Addiction 2007;102:406-12.

20 Macleod J, Whittaker A, Robertson JR. Changes in opiate treatment during attendance at a community drug service-findings from a clinical audit. Drug Alcohol Rev 1998;17:19-25.

21 Strang J, Sheridan J, Hunt C, Kerr B, Gerada C, Pringle M. The prescribing of methadone and other opioids to addicts: national survey of GPs in England and Wales. Br J Gen Pract 2005;55:444-51.
22 Strang J, Hall W, Hickman M, Bird SM. Impact of supervised methadone consumption on deaths related to methadone overdose (1993-2008): analyses using OD4 index in England and Scotland. BMJ 2010;341:c4851.

23 Buster MC, van Brussel GH, van den Brink W. An increase in overdose mortality during the first 2 weeks after entering or re-entering methadone treatment in Amsterdam. Addiction 2002;97:993-1001.

24 Degenhardt L, Randall D, Hall W, Law M, Butler T, Burns L. Mortality among clients of a state-wide opioid pharmacotherapy program over 20 years: risk factors and lives saved. Drug Alcohol Depend 2009;105:9-15.

25 Caplehorn JR, Drummer OH. Mortality associated with New South Wales methadone programs in 1994: lives lost and saved. Med J Aust 1999;170:104-9.

26 Department of Health and the devolved administrations. Drug misuse and dependence: UK guidelines on clinical management. Department of Health, 2007.

27 Davoli M, Bargagli AM, Perucci CA, Schifano P, Belleudi V, Hickman M, et al. Risk of fatal overdose during and after specialist drug treatment: the VEdeTTE study, a national multi-site prospective cohort study. Addiction 2007;102:1954-9.

28 Robertson JR, Raab GM, Bruce M, McKenzie JS, Storkey HR, Salter A. Addressing the efficacy of dihydrocodeine versus methadone as an alternative maintenance treatment for opiate dependence: a randomized controlled trial. Addiction 2006;101:1752-9.

29 Perkins AJ, Kroenke K, Unutzer J, Katon W, Williams JW, Hope C, et al. Common comorbidity scales were similar in their ability to predict health care costs and mortality. / Clin Epidemiol 2004;57:1040-8.

30 Khan NF, Perera R, Harper S, Rose PW. Adaptation and validation of the Charlson index for Read/OXMIS coded databases. BMC Fam Pract 2010;11:1.

31 Hickman M, Carnwath Z, Madden P, Farrell M, Rooney C, Ashcroft R, et al. Drug-related mortality and fatal overdose risk: pilot cohort study of heroin users recruited from specialist drug treatment sites in London. J Urban Health 2003;80:274-87.

32 Ghodse H, Oyefeso A, Kilpatrick B. Mortality of drug addicts in the United Kingdom 1967-1993. Int J Epidemiol 1998;27:473-8.

33 Sweeting MJ, De AD, Ades AE, Hickman M. Estimating the prevalence of ex-injecting drug use in the population. Stat Methods Med Res 2009;18:381-95.

34 Kimber J, Copeland L, Hickman M, Macleod J, McKenzie J, De Angelis D, et al. Survival and cessation in injecting opiate users, prospective observational study of outcomes and the effect of opiate substitute treatment. BMJ 2010;340:c3172.

35 Zador D. Methadone maintenance: making it better. Addiction 2007;102:350-1.

36 Hulse GK, English DR, Milne E, Holman CD. The quantification of mortality resulting from the regular use of illicit opiates. Addiction 1999; $94: 221-9$.

37 Darke S, Zador D. Fatal heroin 'overdose': a review. Addiction 1996;91:1765-72.

38 Farrell M, Marsden J. Acute risk of drug-related death among newly released prisoners in England and Wales. Addiction 2008;103:251-5.

39 McCowan C, Kidd B, Fahey T. Factors associated with mortality in Scottish patients receiving methadone in primary care: retrospective cohort study. BMJ 2009;338:b2225.

40 White JM, Irvine R. Mechanisms of fatal opioid overdose. Addiction 1999;94:961-72.

41 McLellan AT, Lewis DC, O’Brien CP, Kleber HD. Drug dependence, a chronic medical illness: implications for treatment, insurance, and outcomes evaluation. JAMA 2000;284:1689-95.

Accepted: 25 August 2010 